ARTIGO ORIGINAL

ISSN 1677-5090

(c) 2015 Revista de Ciências Médicas e Biológicas

\title{
Fatores de risco do baixo peso ao nascer nos municípios de Juazeiro (BA) e Petrolina (PE)
}

\author{
Risk factors of low birth weight in the municipalities of Juazeiro (BA) and Petrolina (PE) \\ Ana Claudia Morais Godoy Figueiredo ${ }^{1 *}$, Simone Seixas da Cruz², Isaac Suzart Gomes Filho ${ }^{3}$, Luise Maria \\ Souza ${ }^{4}$, Johelle de Santana Passos Soares ${ }^{5}$, Géssica Santana Orrico ${ }^{6}$, Josicélia Estrela Tuy Batista ${ }^{6}$ \\ ${ }^{1}$ Doutoranda em Ciências da Saúde/ Epidemiologia. UnB; ${ }^{2}$ Doutora em Saúde Coletiva/Epidemiologia. UEFS. Professor \\ da UFRB; ${ }^{3}$ Doutor em Odontologia/Periodontia. USP. Professor da UEFS; ${ }^{4}$ Mestre em Saúde Coletiva/Epidemiologia. \\ UEFS; ${ }^{5}$ Doutora em Saúde Coletiva/Epidemiologia. Professor .UFBA; ${ }^{6}$ Acadêmica de Enfermagem. UFRB
}

\begin{abstract}
Resumo
Introdução: O baixo peso ao nascer, conforme a Organização Mundial de Saúde, é caracterizado como peso inferior a 2500 gramas. $O$ evento em questão é considerado um importante determinante da morbimortalidade infantil no Brasil e no mundo, além de predispor a criança a infecções, retardo de crescimento, déficit neuropsicológico pós-natal e baixo desempenho escolar. Objetivo: avaliar a associação entre características maternas e baixo peso ao nascer. Metodologia: Realizou-se uma investigação do tipo caso-controle, em dois hospitais nos municípios vizinhos, Petrolina (PE) e Juazeiro (BA). O tamanho da amostra foi de 588 puérperas, sendo 136 do grupo caso (mulheres com filhos de baixo peso) e 452 do grupo controle (mães de filhos com peso normal). Nos procedimentos de análise de dados avaliou-se distribuição das variáveis, empregando-se o teste qui-quadrado, ao nível de significância de $5 \%$. Este trabalho tem aprovação do Comitê de Ética e Pesquisa da UEFS. Resultados: Houve associação entre ocupação materna durante a gestação $(p=0,04)$, nível de escolaridade paterna $(p=0,01)$, raça/cor da mãe $(p=0,00)$, município de procedência ( $p$ $=0,01)$, idade materna $(p=0,00)$, número de consultas pré-natal $(p=0,00)$, presença do cartão da gestante no parto $(p=0,00)$, número de consultas realizadas pelo profissional médico $(p=0,04)$, número de gestações $(p=0,01)$ e idade gestacional $(p=0,00)$ com o desfecho investigado. Conclusão: Os achados reafirmam que é de suma importância à realização de assistência pré-natal de qualidade, desenvolvendo maiores esforços para identificar os fatores associados ao baixo peso ao nascer, tendo em vista a redução da ocorrência dessa condição gestacional indesejável.
\end{abstract}

Palavras-chave: Baixo peso ao nascer. Epidemiologia.

\begin{abstract}
Introduction: Low birth weight is characterized as less than 2500 grams, according to the World Health Organization. It is considered to be an important determinant of infant morbidity-mortality in Brazil and around the world. Low birth weight predisposes children to infection, growth retardation, postnatal neuropsychological deficit and low school performance. Objective: to evaluate the association between maternal characteristics and low birth weight. Methodology: A case-control investigation was conducted in two hospital in neighboring municipalities: Petrolina (PE) and Juazeiro (BA). The sample consisted of 588 puerperae, of whom 136 were in the case group (women with low-weight children) and 452 in the control group (mothers of normal-weight children). In the data analysis procedures, the distribution of the variables was evaluated using the $x^{2}$ test at the significance level of $5 \%$. This study was approved by the Research Ethics Committee of UEFS. Results: Correlations were found between low birth weight was associated with the mother's occupation during pregnancy $(p=0.04)$, father's schooling level $(p=0.01)$, mother's race/color $(p=0.00)$, municipality of origin ( $p=$ $0.01)$, mother's age $(p=0.00)$, number of prenatal consultations $(p=0.00)$, presence of pregnancy card at time of delivery $(p=0.00)$, number of consultations with a medical professional $(p=0.04)$, number of pregnancies $(p=0.01)$ and gestational age $(p=0.00)$ with the outcome investigated. Conclusion: The findings reaffirm that having quality prenatal care is of utmost importance, with greater efforts made towards identifying factors that are associated with Low birth weight, with a view to reducing the occurrence of this undesirable gestational outcome.
\end{abstract}

Keywords: Low birth weight. Epidemiology.

\section{INTRODUÇÃO}

O peso do recém-nascido e a idade gestacional revelam sobre as condições de saúde e hábitos do cotidiano da mãe e podem demonstrar alguns aspectos da qualidade de vida da parturiente ${ }^{1}$. De acordo com a Organização

Correspondência / Correspondence: *Ana Claudia Morais Godoy Figueiredo, End.: Universidade Federal do Recôncavo da Bahia Av. Carlos Amaral, 1015 - Cajueiro, Santo Antônio de Jesus-BA. CEP: 44.570-000, Email: aninha_m_godoy@hotmail.com
Mundial da Saúde (OMS), todo nascido vivo com peso ao nascer inferior a 2.500 gramas, é considerado de baixo peso ao nascer (BPN), ao passo que é considerado prematuro quando apresenta idade gestacional inferior a 37 semanas.

A prematuridade/baixo peso ao nascer (PBPN) predispõe a criança a infecções, retardo de crescimento, déficit neuropsicológico pós-natal e baixo desempenho escolar². O evento supracitado é um importante preditor da mortalidade infantil e por isso existe um esforço mundial para redução desse indicador ${ }^{1 .}$ 
Conforme os dados oficiais, no Brasil, no ano de 2007, $8,2 \%$ dos nascidos vivos apresentaram peso ao nascer abaixo de 2500 gramas. Na região Nordeste, no mesmo ano, ocorreu $7,45 \%$ de baixo peso, considerada, com essa proporção, a segunda região do país com menor número de nascimentos do evento em questão ${ }^{3}$.

No estado da Bahia, nesse mesmo ano, aproximadamente $8 \%$ dos nascidos vivos possuíam BPN, e em $84,16 \%$ a prematuridade estava presente. Em Salvador, por sua vez, ocorreu 10,17\% de baixo peso. Em Juazeiro, registrou-se que $7,8 \%$ dos nascidos vivos foram categorizados com o mesmo desfecho ${ }^{3}$.

Em Pernambuco, 7,56\% dos nascimentos foram de baixo peso, sendo que cerca de $85 \%$ foram pré-termos, no ano de 2007. Na capital desse estado, cidade de Recife, $8,66 \%$ dos nascidos vivos apresentaram peso inferior a 2500g. Em Petrolina - PE, município do Sub-médio do Vale do São Francisco, esse indicador infantil foi semelhante ao observado em Recife, sendo que $8,83 \%$ das crianças nasceram abaixo do peso ${ }^{3}$.

Existem vários fatores que podem influenciar o fator estudado, como, por exemplo, as características sociodemográficas, biológicas, história gestacional, hábitos de vida da mãe e qualidade da assistência ao pré-natal.

A realização de um pré-natal de qualidade é primordial para a promoção do bem-estar da gestante e do feto. Durante as consultas pré-natal, pode-se identificar, por exemplo: hábitos de vida indesejáveis como tabagismo, consumo excessivo de álcool e alimentação inadequada. Desse modo, é possível desenvolver ações com intuito de minimizar seus efeitos. Além disso, podem ser detectados problemas de saúde como diabetes gestacional, anemia, retardo do crescimento intrauterino, hipertensão arterial sistêmica e infecção do trato urinário. Os fatores supracitados contribuem para nascidos vivos com peso inferior a $2500 \mathrm{~g}$ e podem gerar graves complicações ao feto, tornando-o mais vulnerável à deficiência neuromotora e pulmonar ${ }^{4}$.

Ao lado dos fatores biológicos, encontram-se as características sociodemográficas com alguns determinantes clássicos como renda, nível de escolaridade, idade, etnia, estado civil, condições de moradia, entre outros, que compõem a "teia causal" do BPN".

Considerando-se que existe um complexo de fatores associados ao baixo peso ao nascer que sofre influências regionais, o que dificulta o entendimento do seu processo causal, justifica-se a realização de estudos que demandam esforços para compreender quais e como se comportam os determinantes do referido desfecho. $\mathrm{O}$ objetivo dessa investigação foi avaliar a associação entre as características maternas e o baixo peso ao nascer, em duas unidades hospitalares de municípios vizinhos, Juazeiro-BA e Petrolina-PE.

\section{METODOLOGIA}

\section{Delineamento do estudo}

Foi realizado um estudo do tipo caso-controle exploratório no Hospital Geral Pró-Matre em Juazeiro-BA e Hospital Dom Malan em Petrolina-PE. O grupo caso foi constituído por mulheres cujos filhos apresentaram, ao nascer, peso menor que $2500 \mathrm{~g}$ e/ou idade gestacional menor que 37 semanas. $O$ grupo controle foi formado por mulheres com filhos que tiveram peso ao nascer maior ou igual a $2500 \mathrm{~g}$ e idade gestacional igual ou superior a 37 semanas.

\section{Contexto do estudo}

O Hospital Geral Pró-Matre é uma instituição filantrópica, que realiza atendimentos da rede particular e rede pública. Nesta unidade, a pesquisa foi realizada, apenas, por mulheres atendidas pelo serviço público de saúde.

O Hospital Dom Malan é uma instituição pública que atende, exclusivamente pelo SUS. É uma entidade referência para o cuidado materno-infantil, a assistência é prestada a mulheres, em trabalho de parto, e crianças, em situações de risco e atualmente é administrado pelo Instituto Materno Infantil de Pernambuco (IMIP).

\section{Etapas de investigação}

\section{População alvo e processo de amostragem}

O público alvo foram puérperas atendidas no Hospital Geral Pro-Matre em Juazeiro-BA e no Hospital Dom Malan em Petrolina-PE.

O tamanho mínimo da amostra foi de pelo menos 103 casos e 103 controles para composição da amostra. Para essa fase, empregou-se o programa Epi Info, admitindo-se um nível de confiança $(\alpha)$ de $95 \%$ e um poder do estudo ( $\beta$ ) de $80 \%$, fundamentado no estudo de Cruz et al. ${ }^{6}$.

\section{Critérios de elegibilidade}

Foram selecionadas mulheres na fase puerperal atendidas no Hospital Geral Pro-Matre em Juazeiro-BA e no Hospital Dom Malan no município de Petrolina-PE, com filhos nascidos vivos e com dados de peso e/ou idade gestacional disponíveis em prontuário dos recém-nascidos ou na declaração de nascidos vivos. Participaram do estudo as mães que estiveram presentes no hospital até sete dias, após o parto e concordaram em participar da pesquisa.

\section{Procedimentos de coleta de dados}

Durante um ano e quatro meses foi realizada, diariamente, a seleção das participantes dos grupos caso e controle, conforme os critérios de elegibilidade. As informações de peso e idade gestacional foram adquiridas no prontuário dos recém-nascidos ou na declaração de nascidos vivos.

A coleta de dados se processou, após a puérpera concordar em participar da pesquisa, assinando o Termo de Consentimento Livre e Esclarecido, de acordo com a Resolução $n$ ㅇ 466, de 12 de dezembro de 2012. Em seguida, houve aplicação do questionário, realizada mediante entrevista, executada pelo operador devidamente treinado.

\section{Instrumentos de coleta de dados}

$\mathrm{O}$ instrumento de coleta de dados foi representado por um questionário, que explora os seguintes eixos 
temáticos:

1. Características sociodemográficas

2. Antecedentes Gestacionais

3. Enfermidades Gestacionais

4. Estilo de vida

5. Condição odontológica

\section{Procedimentos de análise de dados}

Inicialmente, foi procedida a avaliação da distribuição de todos os fatores estudados segundo casos e controles. Posteriormente, foram estimadas as medidas de odds ratio e seus respectivos intervalos de confiança a $95 \%$. Nessa etapa, foi empregado o programa estatístico STATA 10.

\section{Aspectos Éticos}

As mulheres que concordaram em participar da pesquisa foram informadas sobre o objetivo, e assinaram o Termo de Consentimento Livre e Esclarecido, de acordo com Resolução 466/2012 sobre os Aspectos Éticos em
Pesquisa com Seres Humanos do Conselho Nacional de Saúde. ${ }^{7}$

O projeto de pesquisa intitulado "Relação entre Doença Periodontal em Gestantes e Nascidos prematuros e/ ou de baixo peso", no qual essa investigação esta inserida tem aprovação do Comitê de Ética em Pesquisa (CEP) da Universidade Estadual de Feira de Santana (UEFS), sob o protocolo n. 152/2008.

\section{RESULTADOS}

Os resultados demonstram que das 588 puérperas, $23,12 \%$ compuseram o grupo caso e $76,88 \%$, o grupo controle. De acordo com a Tabela 1, que apresenta a relação do baixo peso ao nascer com as variáveis sociodemográficas, pode-se observar que a idade das mulheres se distribuiu de forma heterogênea para os grupos caso e controle, com o estrato de mulheres nos extremos da vida reprodutiva mais frequente entre mães de filho com baixo peso ao nascer. Essa diferença foi estatisticamente significante $(p=0,00)$.

Tabela 1 - Características sociodemográficas segundo casos e controles e respectivo p-valor, Hospital Geral Pro-Matre, Juazeiro e Hospital Dom Malan, Petrolina (2009/2010)

\begin{tabular}{|c|c|c|c|c|c|}
\hline \multicolumn{3}{|c|}{ Características Caso (136) } & \multicolumn{2}{|c|}{ Controle (452) } & \multirow[t]{2}{*}{ Valor de $p$} \\
\hline & $\mathbf{N}$ & $\%$ & $\mathbf{N}$ & $\%$ & \\
\hline Idade Materna & & & & & 0,00 \\
\hline$\leq 19$ anos e $\geq 35$ anos & 59 & 43,38 & 32 & 29,20 & \\
\hline$>19$ e $<35$ anos & 77 & 56,62 & 320 & 70,80 & \\
\hline Nível de escolaridade materna & & & & & 0,55 \\
\hline $0-4$ & 112 & 82,35 & 362 & 80,09 & \\
\hline$>4$ anos & 24 & 17,65 & 90 & 19,91 & \\
\hline Renda Familiar & & & & & 0,22 \\
\hline$\leq 1$ salário mínimo & 97 & 71,32 & 297 & 65,71 & \\
\hline > 1 salário mínimo & 39 & 28,68 & 155 & 34,29 & \\
\hline Situação conjugal & & & & & 0,45 \\
\hline Sem companheiro & 27 & 19,85 & 77 & 17,04 & \\
\hline Com companheiro & 109 & 80,15 & 375 & 82,96 & \\
\hline Ocupação da mãe antes da gestação & & & & & 0,04 \\
\hline Atividade sem remuneração & 72 & 52,94 & 194 & 43,02 & \\
\hline Atividade remunerada & 64 & 47,06 & 257 & 56,98 & \\
\hline Ocupação da mãe durante a gestação & & & & & 0,16 \\
\hline Atividade sem remuneração & 100 & 73,53 & 303 & 67,18 & \\
\hline Atividade remunerada & 36 & 26,47 & 148 & 32,82 & \\
\hline Nível de escolaridade paterna & & & & & 0,01 \\
\hline $0-4$ & 100 & 73,53 & 283 & 62,61 & \\
\hline$>4$ anos & 36 & 26,47 & 169 & 37,39 & \\
\hline Ocupação Paterna & & & & & 0,70 \\
\hline Atividade sem remuneração & 66 & 48,53 & 211 & 46,68 & \\
\hline Atividade remunerada & 70 & 51,47 & 241 & 53,32 & \\
\hline Sexo & & & & & 0,66 \\
\hline Masculino & 69 & 50,74 & 239 & 52,88 & \\
\hline Feminino & 67 & 49,26 & 213 & 47,12 & \\
\hline Raça do recém-nascido* & & & & & 0,16 \\
\hline Parda/Preto & 97 & 72,93 & 350 & 78,65 & \\
\hline Branco/outros & 36 & 27,07 & 95 & 21,35 & \\
\hline Raça da mãe* & & & & & 0,00 \\
\hline
\end{tabular}




\begin{tabular}{|c|c|c|c|c|c|}
\hline \multicolumn{3}{|c|}{ Características Caso (136) } & \multicolumn{2}{|c|}{ Controle (452) } & \multirow[t]{2}{*}{ Valor de $\mathrm{p}$} \\
\hline Parda/Preto & 106 & 79,10 & 396 & 89,39 & \\
\hline Branco/outros & 28 & 20,90 & 47 & 10,61 & \\
\hline Número de Moradores em domicilio & & & & & 0,48 \\
\hline $0-4$ & 39 & 28,68 & 144 & 31,86 & \\
\hline$>4$ moradores & 97 & 71,32 & 308 & 68,14 & \\
\hline \multicolumn{6}{|l|}{ Número de filhos } \\
\hline$<2$ filhos & 60 & 44,12 & 208 & 46,02 & 0,69 \\
\hline$\geq 2$ filhos & 76 & 55,88 & 244 & 53,98 & \\
\hline Município de Procedência & & & & & 0,01 \\
\hline Petrolina e Juazeiro & 86 & 63,70 & 354 & 78,32 & \\
\hline Outros & 49 & 36,30 & 98 & 21,68 & \\
\hline
\end{tabular}

Obs: $\mathrm{N}=588$

*Algumas observações foram perdidas por falta de informação acerca da variável

Ao observar a variável ocupação materna antes da gestação, o grupo caso apresentou maior proporção de atividades sem remuneração, sendo essa diferença estatisticamente significante $(p=0,04)$. Entretanto, para ocupação materna não remunerada durante o período gestacional não houve associação significativa, apesar da proporção do grupo caso $(73,53 \%)$ ser maior que a do grupo controle (67,18\%). O nível de escolaridade paterna associou-se com o BPN $(p=0,01)$, níveis de escolaridade inferiores a 4 anos de estudo foi mais frequente no grupo de mulheres com filhos de baixo peso.

Os achados indicam que a variável município de procedência $(p=0,01)$ associou-se ao BPN, observou-se que aproximadamente $36,30 \%$ das mulheres do grupo caso não residiam nos municípios de Petrolina ou Juazeiro. $E$ $21,68 \%$ das mulheres do grupo controle, residiam também em outros municípios. Dentre as 588 participantes da pesquisa, $74,82 \%$ residiam no complexo urbano Petrolina e Juazeiro.

A Tabela 2 apresenta as variáveis da história gestacional das mulheres participantes da pesquisa. Ao analisar principais enfermidades que podem acometer as mulheres, no período gestacional, pode-se observar que apenas houve associação estatisticamente significante para a Hipertensão Arterial Sistêmica $(p=0,00)$, em que o grupo caso $(30,15 \%)$ teve maior proporção para essa doença em relação ao controle $(10,42 \%)$.

Tabela 2 - Características da história gestacional (n e \%) segundo casos e controles e respectivo p-valor. Hospital Geral Pro-Matre, Juazeiro-BA e Hospital Dom Malan, Petrolina (2009/2010)

\begin{tabular}{|c|c|c|c|c|c|}
\hline \multirow{2}{*}{$\begin{array}{l}\text { Características } \\
\text { Hipertensão Arterial }\end{array}$} & \multicolumn{2}{|c|}{ Caso (136) } & \multicolumn{2}{|c|}{ Controle (452) } & \multirow{2}{*}{$\begin{array}{c}\text { Valor de } \boldsymbol{p} \\
0,00\end{array}$} \\
\hline & $N$ & $\%$ & $N$ & $\%$ & \\
\hline Sim & 41 & 30,15 & 47 & 10,42 & \\
\hline Não & 95 & 69,85 & 404 & 89,88 & \\
\hline Infecção Urinária & & & & & 0,78 \\
\hline Sim & 52 & 39,10 & 184 & 41,16 & \\
\hline Não & 81 & 60,90 & 262 & 58,84 & \\
\hline Diabetes & & & & & 0,36 \\
\hline Sim & 2 & 1,49 & 3 & 0,67 & \\
\hline Não & 132 & 98,51 & 446 & 99,13 & \\
\hline Parasitose & & & & & 0,88 \\
\hline Sim & 3 & 2,24 & 11 & 2,45 & \\
\hline Não & 131 & 97,60 & 438 & 97,55 & \\
\hline Epilepsia & & & & & 0,92 \\
\hline Sim & 1 & 0,75 & 3 & 0,67 & \\
\hline Não & 133 & 99,25 & 446 & 99,33 & \\
\hline Pré-Eclampsia* & & & & & 0,45 \\
\hline Sim & 2 & 0,97 & 1 & 2,33 & \\
\hline Não & 42 & 97,03 & 205 & 97,67 & \\
\hline IMC pré-gestacional & & & & & 0,97 \\
\hline$\geq 18,5$ & 81 & 59,56 & 270 & 59,73 & \\
\hline$<18,5$ & 55 & 40,44 & 182 & 40,27 & \\
\hline Gestação de Alto Risco & & & & & 0,80 \\
\hline Sim & 34 & 25,37 & 108 & 24,32 & \\
\hline
\end{tabular}




\begin{tabular}{|c|c|c|c|c|c|}
\hline \multirow{2}{*}{$\begin{array}{ll} & \text { Características } \\
\text { Não } & \end{array}$} & \multicolumn{2}{|c|}{ Caso (136) } & \multicolumn{2}{|c|}{ Controle (452) } & \multirow[t]{2}{*}{ Valor de $p$} \\
\hline & 100 & 74,63 & 336 & 75,68 & \\
\hline Uso de Antibiótico & & & & & 0,49 \\
\hline Sim & 52 & 38,24 & 158 & 35,03 & \\
\hline Não & 84 & 61,76 & 293 & 64,97 & \\
\hline Uso de Anti-inflamatório* & & & & & 0,73 \\
\hline Sim & 6 & 14,63 & 23 & 11,06 & \\
\hline Não & 35 & 85,37 & 184 & 88,94 & \\
\hline Uso de Sulfato Ferroso & & & & & 0,15 \\
\hline Sim & 95 & 70,37 & 344 & 76,44 & \\
\hline Não & 40 & 29,63 & 106 & 23,56 & \\
\hline Uso de ácido fólico & & & & & 0,01 \\
\hline Sim & 79 & 58,52 & 316 & 70,22 & \\
\hline Não & 56 & 41,48 & 134 & 29,78 & \\
\hline Realização do Pré-Natal & & & & & 0,16 \\
\hline Sim & 117 & 86,03 & 408 & 90,27 & \\
\hline Não & 44 & 13,97 & 19 & 7,93 & \\
\hline Número de consultas pré-natal & & & & & 0,00 \\
\hline$<6$ consultas & 81 & 59,56 & 160 & 35,40 & \\
\hline$\geq 6$ consultas & 55 & 40,44 & 292 & 64,60 & \\
\hline $\begin{array}{l}\text { Presença do Cartão da gestante no } \\
\text { hospital }\end{array}$ & & & & & 0,00 \\
\hline Sim & 104 & 76,47 & 394 & 87,17 & \\
\hline Não & 31 & 23,53 & 57 & 12,83 & \\
\hline Início do Pré-Natal & & & & & 0,10 \\
\hline$\leq 16$ semanas & 95 & 69,85 & 347 & 76,77 & \\
\hline$>16$ semanas & 41 & 30,15 & 105 & 23,23 & \\
\hline Número de Consultas Médicas & & & & & 0,04 \\
\hline$\geq 3$ & 34 & 25,00 & 155 & 34,29 & \\
\hline$<3$ & 102 & 75,00 & 297 & 65,71 & \\
\hline Vacinação antitetânica & & & & & 0,73 \\
\hline$<2$ doses & 33 & 24,44 & 116 & 25,89 & \\
\hline$\geq 2$ doses & 102 & 75,56 & 332 & 74,11 & \\
\hline Primiparidade & & & & & 0,01 \\
\hline Sim & 4 & 2,94 & 2 & 0,44 & \\
\hline Não & 132 & 97,06 & 450 & 99,56 & \\
\hline Nascimento de baixo peso anterior & & & & & 0,28 \\
\hline Sim & 14 & 10,37 & 34 & 7,52 & \\
\hline Não & 121 & 89,63 & 418 & 92,48 & \\
\hline Idade Gestacional & & & & & 0,00 \\
\hline$<37$ semanas & 50 & 36,76 & 103 & 22,79 & \\
\hline$\geq 37$ semanas & 86 & 63,24 & 349 & 77,21 & \\
\hline
\end{tabular}

Obs: $\mathrm{N}=588$

*Variáveis avaliadas apenas na amostra de Juazeiro-BA, visto que estas foram incluídas após coleta de dados de Petrolina-PE.

Para a ausência de ácido fólico durante a gestação, número de consultas pré-natal, presença do cartão da gestante no momento do parto e número de consultas médicas realizadas na gravidez, houve forte associação estatística. Outro fator que se associou ao baixo peso ao nascer, foi o número de gestações, a frequência relativa das mulheres que tiveram mais de dois filhos do grupo caso $(97,06 \%)$ foi inferior àquela encontrada para o grupo sem o desfecho $(99,56 \%)$, sendo altamente significante, esta associação $(p=0,01)$.

Ressalta-se ainda que para a variável baixo peso anterior não houve relação relevante com o BPN, todavia as mulheres do grupo de mães com filhos de baixo peso apresentaram cerca de $3 \%$ a mais, de nascimentos prévios com baixo peso. A primiparidade também se mostrou associada ao BPN $(p=0,01)$.

Neste estudo, observou-se que a idade gestacional inferior a 37 semanas, esteve associada ao BPN, $(p=0,00)$. Pode-se observar que nas puérperas do grupo caso houve uma diferença de aproximadamente de $14 \%$, quando confrontado ao grupo controle.

Ao avaliar os fatores relacionados à saúde bucal, observou-se que as puérperas do grupo caso realizaram menor número proporcional de visitas ao dentista, não 
havendo diferença estatística entre os grupos $(p=0,11)$.

relato do uso do fio dental durante a gestação $(p=0,55)$.

Essa mesma tendência foi detectada também para o

Tabela 3 - Características de estilo de vida (n e \%) segundo casos e controles e respectivo p-valor. Hospital Geral Pro-Matre, Juazeiro-BA e Hospital Dom Malan, Petrolina-PE (2009/2010)

\begin{tabular}{|c|c|c|c|c|c|}
\hline \multirow{2}{*}{$\begin{array}{l}\text { Características } \\
\text { Hábito de Fumar }\end{array}$} & \multicolumn{2}{|c|}{ Caso (136) } & \multicolumn{2}{|c|}{ Controle (452) } & \multirow{2}{*}{$\begin{array}{c}\text { Valor de } p \\
0,69\end{array}$} \\
\hline & $\mathbf{N}$ & $\%$ & $\mathbf{N}$ & $\%$ & \\
\hline Sim & 47 & 34,56 & 148 & 32,74 & \\
\hline Não & 89 & 65,44 & 304 & 67,26 & \\
\hline \multicolumn{6}{|l|}{ Consumo de bebida alcoólica } \\
\hline 0 gramas & 66 & 48,89 & 212 & 47,01 & 0,24 \\
\hline$<14$ gramas & 68 & 50,37 & 219 & 48,56 & \\
\hline 14 a 27 gramas & 0 & 0,00 & 2 & 0,44 & \\
\hline$\geq 28$ gramas & 1 & 0,74 & 18 & 3,99 & \\
\hline Droga & & & & & 0,95 \\
\hline Sim & 2 & 1,49 & 7 & 1,56 & \\
\hline Não & 132 & 98,51 & 442 & 98,44 & \\
\hline \multicolumn{6}{|l|}{ Uso de fio dental } \\
\hline Sim & 101 & 74,26 & 324 & 71,68 & 0,55 \\
\hline Não & 35 & 25,74 & 128 & 28,32 & \\
\hline \multicolumn{6}{|l|}{ Visita ao dentista } \\
\hline Sim & 97 & 63,94 & 289 & 71,32 & 0,11 \\
\hline Não & 39 & 36,06 & 163 & 28,68 & \\
\hline
\end{tabular}

Obs.: $\mathrm{N}=588$

Neste estudo, observou-se que houve associação com o BPN para os seguintes fatores:

A idade materna associou-se ao BPN neste estudo. As mães com menos de 19 anos são mais vulneráveis a terem filhos de baixo peso ao nascer, devido a imaturidade biológica do organismo da mulher que pode promover o desenvolvimento fetal inadequado. Já as mulheres acima de 35 anos podem ter filhos de baixo peso, devido à possível presença de doenças maternas pré-existentes que aumenta com a idade, alguns estudos ratificam esses achados $^{8,9}$.

O nível de escolaridade materna, aqui, não esteve associado ao desfecho baixo peso ao nascer, corroborando com os estudos de Medeiros e Gouveia e Geib e colaboradores ${ }^{10,11}$. No entanto, alguns estudos apontam que essa variável pode influenciar no BPN, visto que as mulheres que tem menos anos de estudo, são aquelas com as piores condições socioeconômicas, e assim, sentem mais dificuldade para a realização dos cuidados com o binômio mãe-feto durante a gestação ${ }^{12,13,14}$.

No que se refere à ocupação e nível de escolaridade paterna se observou associação ao BPN, neste estudo, ratificando outros achados ${ }^{15}$. Alguns estudos indicam a associação entre as variáveis supracitadas e o baixo peso $^{16,17}$. Conforme Audi e colaboradores ${ }^{18}$, as mulheres, cujos companheiros trabalham em categorias profissionais que exigem menor nível de escolaridade, a exemplo do setor agropecuário, são mais propensas a terem filhos com BPN.

Neste estudo, não houve associação entre o estado civil e o BPN, confirmando o estudo de Li e colabora- dores ${ }^{12}$. Entretanto, a condição conjugal, de acordo com Rocha ${ }^{19}$ pode influenciar no baixo peso ao nascer, tendo em vista que as mulheres solteiras que, no mais das vezes, sustentam e cuidam dos seus filhos, além de realizarem as atividades de casa, sem o apoio de um companheiro, despendem menos atenção com saúde durante a gestação.

As atividades não remuneradas antes da gestação relacionaram-se com o BPN, neste estudo coincidi com outros pesquisadores ${ }^{13}$ quando afirmam que mulheres que não trabalham fora do lar, tendem a ter um padrão socioeconômico familiar pouco favorável, e assim menos recursos com os cuidados em saúde, alimentação e moradia.

A raça/cor materna não se associou ao desfecho BPN. Todavia, alguns estudos apontam que as mulheres negras comumente pertencem ao estrato socioeconômico mais desfavorável, e assim tornam-se mais susceptíveis a terem filhos com baixo peso ao nascer2, 14, 20-21.

Em relação à assistência pré-natal, a ausência do cartão da gestante durante a hospitalização para o parto, o número de consultas pré-natal, número de consultas com o profissional médico houve associação com o desfecho, neste estudo. Na tentativa de explicar tais associações, alguns estudos apontam que o número e a qualidade das consultas pré-natal são de suma importância para o bom desenvolvimento da gestação, 20,22 . Um trabalho especificamente ${ }^{13}$ afirma que a realização de mais que sete consultas, durante o pré-natal é um fator de proteção para o desfecho em questão. Além disso, a presença do cartão da gestante na ocasião do parto pode representar um comportamento de saúde mais satisfatório em relação 
aos cuidados gestacionais, refletindo uma atitude da muIher durante o período gestacional, mais protetora para o binômio mãe-filho.

Sabe-se que uso de ácido fólico é de suma importância para o bom desenvolvimento fetal, pois colabora na formação do sistema neurológico da criança durante a gestação ${ }^{4}$. Neste estudo, o ácido fólico foi considerado um fator de proteção para o BPN corroborando com a pesquisa de Jafari e colaboradoes ${ }^{15}$.

O número de nascimentos prévios com baixo peso ao nascer não esteve associado ao desfecho, nesta pesquisa. É possível que esse dado tenha sido distorcido, vez que era necessário um recordatório da mãe sobre o peso de filhos de gestações anteriores. Pois, a literatura demonstra que as mulheres com filhos de baixo peso em gestações anteriores, têm maiores chances do evento se repetir no futuro ${ }^{18,20}$. As mulheres com filhos que nascem com baixo peso, devem ter uma assistência pré-natal diferenciada com o intuito de reduzir o desfecho BPN.

A gestação de alto risco também não esteve associada ao baixo peso ao nascer neste estudo, embora a literatura aponte para essa direção ${ }^{4,14,23}$. Assim, desconfia-se também que pode ter havido distorção acerca dessa variável no estudo.

Embora nos resultados dessa pesquisa, não foi encontrado associação entre a maioria das enfermidades maternas e o baixo peso ao nascer, a Hipertensão Arterial Sistêmica influenciou o baixo peso ao nascer de forma relevante. A hipertensão arterial determina o baixo peso ao nascer, visto que a doença supracitada pode acelerar o trabalho de parto, causando assim a prematuridade e o baixo peso ao nascer ${ }^{14,20 .}$

Visto que a infecção do trato urinário e a pré-eclâmpsia são fatores clássicos para o BPN, pode-se suspeitar de que os diagnósticos dessas doenças estejam sendo realizados de forma insatisfatória. A infecção urinária pode trazer graves consequências ao feto, visto que a enfermidade pode afetar o sistema neurológico e respiratório da criança $^{4,23,24}$.

No referido estudo, o índice de massa corpórea pré-gestacional, demonstra a relação de peso e altura materna, também não se associou ao baixo peso ao nascer, corroborando com o estudo de Silva e outros ${ }^{20}$. Embora, o IMC, inferior a 18,5, indica que o peso da gestante é inadequado para a gestação o que pode contribuir para restrição do crescimento fetal ${ }^{25}$.

Em relação às variáveis de estilo de vida, nessa pesquisa, não houve associação entre hábito de fumar, consumo de álcool, não uso de fio dental e ausência de visita ao dentista com o baixo peso ao nascer. Pode-se suspeitar que houve distorção da realidade pelas respondentes no que se refere a hábito de fumar e consumo de álcool nessa fase da vida, visto que esses são considerados hábitos deletérios e muito prejudicial a saúde e veiculados pela mídia comum com danosos ao feto. Dado que, o consumo de mais de $12 \mathrm{~g}$ de álcool e/ou qualquer uso de cigarros na gestação, são prejudiciais ao feto, e pode contribuir para o parto prematuro e BPN ${ }^{26}$.

Assim, a principal limitação diz respeito ao registro das informações obtidas, bem como da validação de alguns dados colhidos por entrevistas. Particularmente, aqueles que dependem da memória e da boa vontade da informante, havendo possibilidade de viés recordatório, visto que algumas mães podem ter esquecido ou omitido alguns dados durante a entrevista.

\section{CONCLUSÃO}

Os resultados demonstram que se associaram ao baixo peso ao nascer as variáveis: a idade materna, ocupação da mãe antes da gravidez, nível de escolaridade paterna, raça da mãe, município de procedência, número de consultas pré-natal, número de consultas pré-natal com o profissional médico, uso de ácido fólico, presença do cartão da gestante, paridade, hipertensão arterial, prematuridade e número de gestações.

Dessa forma, os achados demonstram a importância da realização do que é preconizado pelo Ministério da Saúde para assistência pré-natal de qualidade, com a captação precoce da gestante, realização de no mínimo seis consultas pré-natal, acompanhamento do estado de saúde-doença da mulher durante a gestação, identificando assim as enfermidades e hábitos de vida que podem influenciar no baixo peso ao nascer. Por outro lado, na população estudada, essas não parecem ser recomendações fáceis de serem seguidas, havendo necessidade de planejar novas ações para atingir essas metas determinadas pelo Ministério da Saúde.

\section{AGRADECIMENTOS}

À Fundação de Amparo à Pesquisa na Bahia (FAPESB) que financiou a investigação no município de Juazeiro-BA e à Fundação de Amparo à Ciência e Tecnologia do Estado de Pernambuco (FACEPE) que financiou a pesquisa em Petrolina-PE.

\section{REFERÊNCIAS}

1. ARAÚJO, D. M. R.; PEREIRA, N. D. L.; KAC, G. Ansiedade na gestação, prematuridade e baixo peso ao nascer: uma revisão sistemática da literatura. Cad. Saúde Pública, Rio de Janeiro, v. 23, n. 4, p. 747-756, 2007.

2. ARAÚJO, B. F. D.; TANAKA, A. C. D. A. Fatores de risco associados ao nascimento de recém-nascidos de muito baixo peso em uma população de baixa renda. Cad. Saúde Pública, Rio de Janeiro, v. 23, n. 12, p. $2869-$ 2877, 2007.

3. BRASIL. Ministério da Saúde. DATASUS. Informações relacionadas à saúde: indicadores de saúde (nascidos vivos), 2007. Brasília: Ministério da Saúde, 2007. Disponível em: < http://tabnet.datasus.gov.br/cgi/ idb2007/c01.htm >. Acesso em: 20 mar. 2010.

4.

4. ㄴ. Secretaria de Atenção à Saúde. Departamento de Ações Programáticas Estratégicas. Área Técnica de Saúde da Mulher. Pré-natal e Puerpério: atenção qualificada e humanizada - manual técnico. Brasília: Ministério da Saúde, 2012. 53 p.

5. VALERO DE BERNABE, J. et al. Risk factors for low birth weight: a review. Eur. j. obstet. gynecol. reprod. biol., Amsterdam, v. 116, n. 1, p. 3-15, 2004. 
6. CRUZ, S. S. et al. Periodontal therapy for pregnant women and cases of low birthweight: an intervention study. Pediatr. int., Carlton South, v. 52 , n. 1 , p. 57-64, 2010.

7. BRASIL. Conselho Nacional de saúde. Resolução no 466, de 2012. Diretrizes e normas regulamentadoras de pesquisas envolvendo seres humanos. Brasília, DF, Diário Oficial da União, v. 12, Seção 1. p. 59, jun. 2013.

8. SU, P. H. et al. Transthyretin levels are not related to Apgar score in low birth weight and very low birth weight infants. Early hum. dev., Amsterdam, v. 84, n. 8, p. 533-538, ago, 2008.

9. KHASHAN, A. S.; BAKER, P. N.; KENNY, L. C. Preterm birth and reduced birthweight in first and second teenage pregnancies: a register-based cohort study. BMC pregnancy childbirth, London, v. 10, doi:10.1186/1471-2393-10-36, 2010.

10. MEDEIROS, A.; GOUVEIA, N. Relação entre baixo peso ao nascer e a poluição do ar no Município de São Paulo. Rev. Saúde Pública, São Paulo, v. 39, n. 6, p. 965-972, 2005.

11. GEIB, L. T. C. et al. Determinantes sociais e biológicos da mortalidade infantil em coorte de base populacional em Passo Fundo, Rio Grande do Sul. Ciênc. saúde coletiva., Rio de Janeiro, v. 15, n. 2, p. 363-370, 2010.

12. LI, C. Y.; SUNG, F. C. Socio-economic inequalities in low-birth weight, full-term babies from singleton pregnancies in Taiwan. Public health, London, v. 122, n. 3, p. 243-250, 2008.

13. CARNIEL, E. D. F. et al. Determinantes do baixo peso ao nascer a partir das Declarações de Nascidos Vivos. Rev. bras. epidemiol., São Paulo, v. 11, n. 1, p. 169-179, 2008.

14. JESSE, D. E. et al. Racial disparities in biopsychosocial factors and spontaneous preterm birth among rural low-income women. J. midwifery womens health., New York, v. 54, n. 1, p. 35-42, 2009.

15 JAFARI, F. et al. Socio-economic and medical determinants of low birth weight in Iran: 20 years after establishment of a primary healthcare network. Public health., London, v. 124, n. 3, p. 153-158, 2010.

16 WANG, W. L. et al. Low birth weight, prematurity, and paternal social status: impact on the basic competence test in Taiwanese adolescents.
J. pediatr., St. Louis, v. 153, n. 3, p. 333-338, 2008.

17. MU, S. C. et al. Cognitive development at age 8 years in very low birth weight children in Taiwan. J. Formos. Med Assoc., Taipei, v. 107, n. 12, p. 915-920, 2008.

18. AUDI, C. A. F. et al. Associação entre violência doméstica na gestação e peso ao nascer ou prematuridade. J. pediatr., Porto Alegre, v. 84, n. 1, p. 60-67, 2008.

19. ROCHA, R. C. et al. Prematurity and low birth weight among Brazilian adolescents and young adults. J. Pediatr. Adolesc. Gynecol., Philadelphia, v. 23, n. 3, p. 142-145, 2010.

20. SILVA, A. M. R. et al. Fatores de risco para nascimentos pré-termo em Londrina, Paraná, Brasil. Cad. Saúde Pública, Rio de Janeiro, v. 25 n. 10, p. 2125-2138, 2009.

21. SILVA, L. M. et al. Racial inequalities and perinatal health in the southeast region of Brazil. Braz j. med. biol. res., Ribeirão Preto, v. 40, n.9, p. 1187-1194, 2007.

22. SEQUE, C. A. et al. Assistência pré-natal e ao parto de mães de crianças usuárias do serviço de vacinação de unidade de saúde da cidade de São Paulo. Rev. paul. pediatr., São Paulo, v. 25, n. 1, p. 38-46, 2007.

23. SILVEIRA, M. F. et al. Aumento da prematuridade no Brasil: revisão de estudos de base populacional. Rev. saúde pública, São Paulo, v. 42, n. 5, p. 957-964, 2008.

24. NASCIMENTO, L. F. C. Estudo transversal sobre fatores associados ao baixo peso ao nascer a partir de informações obtidas em sala de vacinação. Rev. bras. saúde matern. infant., Recife, v. 3, n. 1, p. 37-42, 2003.

25. PÉREZ GUIRADO, N. M.; PRESNO LABRADOR, C.; SARMIENTO BROOKS, G. Algunos factores de riesgo asociados al recién nacido con bajo peso. Rev. cuba. med. gen. integr., Habana, v. 21, n. 3/4, p. 1-5, 2005

26. MARISCAL, M. et al. Pattern of alcohol consumption during pregnancy and risk for low birth weight. Ann. epidemiol., New York, v. 16, n. 6, p. 432-438, 2006.

Submetido em: 04.12.2014.

Aceito em: 14.03.2015 